\title{
Administración de albúmina a largo plazo en cirrosis descompensada (ANSWER): un estudio abierto aleatorizado
}

\author{
Joaquin Sotomayor B. ${ }^{l}$, Álvaro Ananías S. ${ }^{l}$, Pauline Böhm G. ${ }^{l}$, \\ Andrés Soffia P. ${ }^{l}$, Luis Antonio Díaz P. ${ }^{2}$ y Juan Pablo Arab $V^{2}$
}

\author{
Long-term albumin administration in decompensated cirrhosis (ANSWER): \\ an open-label randomized trial
}

\section{Pregunta clínica}

En pacientes adultos cirróticos con ascitis no complicada que están recibiendo tratamiento con antagonistas de aldosterona ( $\geq 200 \mathrm{mg} /$ día) y furosemida ( $\geq 25 \mathrm{mg} /$ día), ¿Es más eficaz el administrar albúmina humana $(\mathrm{AH})$ junto al tratamiento médico estándar (TME) versus solo el TME?

Para responder a esta pregunta se revisará el siguiente artículo: Caraceni P, Riggio O, Angeli P, Alessandria C, Neri S, Foschi F, Levantesi F, Airoldi $\mathrm{A}$ et al. Long-term albumin administration in decompensated cirrhosis (ANSWER): an open-label randomized trial. Lancet. 2018 16;391:2417-29¹, así como su material suplementario ${ }^{2}$.

\section{Contexto}

La cirrosis descompensada tiene un mal pronóstico clínico, siendo la sobrevida promedio de estos pacientes cercana a los 2 años ${ }^{3}$. Su complicación más frecuente es la aparición de ascitis, es decir, la acumulación patológica de líquido en la cavidad peritoneal dependiente de hipertensión portal, que ha demostrado ser un predictor independiente de mortalidad en este grupo de pacientes ${ }^{4,5}$. En pacientes cirróticos descompensados, el manejo de la ascitis continúa representando un desafío terapéutico y, aunque su único tratamiento definitivo es el trasplante hepático, se han planteado múltiples alternativas para su manejo sintomático.

La administración a largo plazo de albúmina humana $(\mathrm{AH})$ ha sido tema de discusión recurrente en las últimas décadas, probablemente dada la alta frecuencia de hipoalbuminemia en estos pacientes. $\mathrm{Si}$ bien podría resultar intuitivo perseguir la nor- malización de sus valores en miras de aumentar la presión oncótica sanguínea, y de esta forma reducir la aparición y/o desarrollo de ascitis, la hipoalbuminemia per se no pareciera tener un rol patogénico primario en esta enfermedad, ya que la gradiente de presión coloido-osmótica no se encuentra reducida en pacientes cirróticos ${ }^{6}$. Sin embargo, la AH es capaz de aumentar el volumen sanguíneo y la perfusión renal, pudiendo disminuir de forma secundaria la activación de ejes hormonales retenedores de sal y agua característicamente hiperfuncionantes en este escenario ${ }^{7}$. Aunque la administración de albúmina es bien tolerada, puede presentar reacciones alérgicas inmediatas, disminución brusca en la presión arterial y, en pacientes adultos mayores o con factores de riesgo de falla cardíaca, insuficiencia cardíaca congestiva ${ }^{8}$. Por otro lado, no se conoce con certeza el rol de los efectos no-oncóticos de la albúmina (ej. antioxidante, cotransportadora, etc.), que pudieran potenciar su efecto benéfico en pacientes cirróticos 9 .

Los efectos de la administración de $\mathrm{AH}$ junto al TME fueron evaluados previamente a través de dos estudios clínicos prospectivos controlados en pacientes con cirrosis descompensada con ascitis diagnosticada por ultrasonido, ejecutados por un mismo grupo de investigación. En el primer ensayo, la administración de $25 \mathrm{~g}$ de $\mathrm{AH}$ semanales por un año y $25 \mathrm{~g}$ de AH cada 2 semanas durante los siguientes 2 años fue más efectiva en el tratamiento de la ascitis que el uso aislado de diuréticos y redujo la probabilidad de readmisión hospitalaria, sin efectos en la sobrevida ${ }^{10}$. El segundo estudio extendió el seguimiento de los pacientes a una media de 84 meses, reportando una mejoría en la sobrevida libre de trasplante ${ }^{11}$. Sin embargo, el pequeño tamaño de la muestra impide sacar conclusiones sólidas y las guías actuales de manejo no incluyen esta medida terapéutica.
Escuela de Medicina, Facultad de Medicina. Pontificia Universidad Católica de Chile. Santiago, Chile. 2Departamento de Gastroenterología Escuela de Medicina Facultad de Medicina. Pontificia Universidad Católica de Chile. Santiago, Chile.

Recibido:

Aceptado:

Correspondencia a: Dr. Juan Pablo Arab Verdugo

Departamento de Gastroenterología Pontificia Universidad Católica de Chile. Tel. +56223543520 jparab@uc.cl 
En este contexto, el estudio de Caraceni P. et al. pretendió comparar el efecto de administrar a largo plazo AH junto a TME versus solo TME, en términos de mortalidad, manejo de ascitis e incidencia de complicaciones en pacientes con cirrosis descompensada.

\section{Métodos}

\section{Características generales}

Pacientes: Se incluyeron 440 pacientes cirróticos con ascitis no complicada, seleccionados en admisiones hospitalarias y en consultas ambulatorias de 33 hospitales italianos. De estos, nueve pacientes resultaron inelegibles, por lo que 431 pacientes ingresaron al protocolo. Los criterios de inclusión y exclusión están expuestos en la Tabla 1.

Intervención: Los pacientes fueron randomizados a un grupo de intervención y otro de control, a los que durante 18 meses se les administró AH y TME o solo TME, respectivamente. La intervención correspondió a la administración de AH (40 g dos veces por semana por 2 semanas, y luego $40 \mathrm{~g}$ semanales por 18 meses), suministrando TME de acuerdo a las indicaciones de las guías clínicas disponibles ${ }^{11-13}$. El TME consideró la administración de una dieta hiposódica, ajuste de dosis de diuréticos en función de la evolución de la ascitis, eventual paracentesis terapéutica (asociada a administración de albúmina) en caso de ascitis a tensión, sintomática o refractaria a tratamiento y eventual instalación de transjugular intrahepatic portosystemic shunt (TIPS) en caso de sangrado digestivo no controlado y ascitis refractaria. Los tratamientos de peritonitis bacteriana espontánea (PBE) y síndrome hepatorrenal (SHR) tipo 1 incluyeron la administración de albúmina. Además, se realizó un manejo etiológico de los pacientes, administrando tratamiento antiviral en caso de infecciones por virus hepatitis B y C y promoviendo la abstinencia alcohólica ${ }^{2}$. Los pacientes fueron evaluados de forma mensual por 18 meses o hasta la interrupción del estudio o muerte, registrando de forma electrónica datos clínicos, de laboratorio e instrumentales (de ser necesarios). Se incluyeron entrevistas sobre la ingesta dietaria de sodio y alcohol, pudiendo consultar a familiares. La calidad de vida fue valorada con la Escala Visual Análoga (EVA) y el 5-Dimension Self-Report Questionnaire (EQ-5D) ${ }^{14,15}$. Contrariamente a lo planificado en el protocolo original del estudio, no se utilizó el cuestionario Short Form 36, debido a un insuficiente número de formularios completados adecuadamente.

Resultados (outcomes): El outcome primario fue la mortalidad a 18 meses. Los outcomes secundarios fueron: 1) número de paracentesis terapéuticas; 2) dosis acumulada de diuréticos; 3 ) hiponatremia (concentración de sodio sérico $<130 \mathrm{mmol} / \mathrm{L}$ ) o hiperkalemia (concentración de potasio sérico $\geq 5,5 \mathrm{mmol} / \mathrm{L}$; 4) incidencia de complicaciones relacionadas a la cirrosis (ascitis refractaria, PBE, infecciones bacterianas, insuficiencia renal aguda, SHR tipo 1, encefalopatía hepática grado 3 o 4 y hemorragia gastrointestinal relacionada a hipertensión portal); 5) necesidad de 3 o más paracentesis terapéuticas por mes; 6) calidad de vida; 7) número y duración de admisiones hospitalarias y 8) costo-efectividad del tratamiento. Los efectos adversos fueron evaluados a lo largo de todo el estudio, reportando su severidad y relación a $\mathrm{AH}$ de acuerdo a la opinión del investigador.

\section{Evaluación de la validez interna}

Diseño: Estudio clínico multicéntrico, aleatorizado, abierto, paralelo y de tipo pragmático. Se estimaron 210 pacientes por grupo para asegurar un poder estadístico del $90 \%$.

Aleatorización: Los pacientes fueron randomizados a recibir $\mathrm{AH}$ y TME o solo TME, según lo designado en las dos ramas del estudio. La secuencia de aleatorización fue ciega, generada a computador en razón de $1: 1$, a través de una permutación de bloques de cuatro celdas, estratificados según la necesidad de paracentesis terapéutica en el último mes (sí o no) y el valor de la natremia $(<135 \mathrm{mmol} / \mathrm{L} \mathrm{o} \geq 135 \mathrm{mmol} / \mathrm{L})$.

Tabla 1. Criterios de inclusión y exclusión

\section{Criterios de inclusión}

Diagnóstico de cirrosis con ascitis no complicada recibiendo antagonistas de aldosterona ( $\geq 200 \mathrm{mg} /$ día) y furosemida $(\geq 25 \mathrm{mg} /$ día)

Endoscopia digestiva alta en los últimos 12 meses

Ecografía abdominal en los últimos 30 días

Exámenes de laboratorio protocolares en los últimos 7 días

\section{Criterios de exclusión}

$<18$ años

Ascitis refractaria o complicaciones recientes de la cirrosis TIPS

Carcinoma hepatocelular activo

Trasplante hepático

Consumo de alcohol activo

Falla orgánica extrahepática

Uso de AH para el tratamiento de la ascitis en el último mes

TIPS: transjugular intrahepatic portosystemic shunt. AH: albúmina humana. 
Grupos de estudio: La muestra fue balanceada en relación a características demográficas, clínicas y de laboratorio.

Ciego: La secuencia de aleatorización fue generada de forma ciega. Los pacientes, médicos y estadísticos no fueron ciegos al tratamiento asignado.

Seguimiento: Del total de pacientes enrolados inicialmente, que cumplían los criterios de inclusión, se perdió el seguimiento a 30 pacientes, 17 pacientes de la rama $\mathrm{TME}+\mathrm{AH}$ y 13 de la rama $\mathrm{TME}$, correspondiendo al $6,9 \%$ de la muestra.

Escenario: Desarrollado en 33 hospitales italianos académicos y no académicos.

Tipo de análisis: Por intención de tratar modificada, dado que el análisis no incluyó a seis pacientes que se excluyeron dentro de los primeros 30 días de reclutamiento por inclusión errónea ni a tres pacientes que retiraron su consentimiento informado previo al inicio de tratamiento.

Tiempo de seguimiento: Seguimiento de 18 meses o menos si se alcanzaba alguno de los siguientes eventos: trasplante hepático, TIPS, necesidad de tres o más paracentesis por mes, rechazo a continuar su participación o muerte.

Interrupción precoz por beneficio: No hubo.

\section{Resultados principales}

De los 440 pacientes incluidos, nueve resultaron inelegibles y 83 finalizaron el estudio de forma prematura, por lo que 348 pacientes completaron el estudio (176 y 172 en las ramas TME + AH y TME, respectivamente). En relación al outcome primario, la mortalidad a los 18 meses fue significativamente menor en el grupo de pacientes que recibió el esquema de tratamiento TME + AH (Tabla 2).

Con respecto a los outcomes secundarios evaluados, el grupo de pacientes que recibió el esquema de tratamiento $\mathrm{TME}+\mathrm{AH}$ se vio beneficiado de forma estadísticamente significativa en relación al número de paracentesis terapéuticas, eventos de hiponatremia e hiperkalemia, incidencia de complicaciones relacionadas a la cirrosis, necesidad de 3 o más paracentesis terapéuticas por mes e incremento de calidad de vida. Asimismo, se observó una reducción significativa en el número y duración de las admisiones hospitalarias, cuyas principales causas se exponen en la Tabla 3.

Tabla 2. Comparación del outcome primario en grupo TME + AH versus TME

\begin{tabular}{|lccccccc|}
\hline Outcome primario & Grupo TME + AH & Grupo TME & RR & RRR & RAR & NNT & Valor p \\
Mortalidad a 18 meses & 0,27 & 0,44 & 0,61 & 0,39 & 0,17 & 5,88 & 0,037 \\
& {$[$ IC 95\% 0,19-0,37] } & {$[$ IC 95\% 0,32-0,80] } & & & & \\
\hline
\end{tabular}

AH: Albúmina humana. TME: Tratamiento médico estándar. IC: Intervalo de confianza. RR: Riesgo relativo. RRR: Reducción del riesgo relativo. RRA: Reducción del riesgo absoluto. NNT: Número necesario a tratar.

Tabla 3. Principales causas de admisión hospitalaria en grupo TME + AH versus TME

\begin{tabular}{|lcccc|}
\hline $\begin{array}{l}\text { Principales causas de admisión } \\
\text { hospitalaria }\end{array}$ & $\begin{array}{c}\text { Grupo TME + AH } \\
\text { TI [IC 95\%] }\end{array}$ & $\begin{array}{c}\text { Grupo TME } \\
\text { TI [IC 95\%] }\end{array}$ & RTI [IC 95\%] & Valor p \\
\hline Deterioro de la función hepática & $0,06[0,03-0,10]$ & $0,29[0,21-0,38]$ & $0,21[0,10-0,40]$ & $<0,0001$ \\
\hline Ascitis & $0,06[0,03-0,10]$ & $0,13[0,08-0,20]$ & $0,45[0,20-0,96]$ & 0,031 \\
PBE & $0,04[0,02-0,08]$ & $0,10[0,06-0,16]$ & $0,42[0,16-1,01]$ & 0,041 \\
\hline Infección espontánea no PBE & $0,13[0,09-0,19]$ & $0,29[0,21-0,39]$ & $0,56[0,33-0,94]$ & 0,024 \\
Encefalopatía hepática & $0,26[0,20-0,34]$ & $0,36[0,27-0,47]$ & $0,72[0,49-1,06]$ & 0,085 \\
Hemorragia digestiva & $0,11[0,07-0,17]$ & $0,05[0,02-0,10]$ & $2,23[0,97-5,73]$ & 0,049 \\
\hline Admisión para procedimiento agendado & $0,21[0,15-0,28]$ & $0,30[0,22-0,40]$ & $0,71[0,15-0,28]$ & 0,114 \\
\hline
\end{tabular}

AH: Albúmina humana. TME: Tratamiento médico estándar. TI: Tasa de incidencia. IC 95\%: Intervalo de confianza del 95\%. RRR: Reducción del riesgo relativo. RTI: Razón de tasas de incidencia. PBE: Peritonitis bacteriana espontánea. 
Además, se objetivó que el tratamiento $\mathrm{TME}+\mathrm{AH}$ es costo-efectivo por sobre el manejo con TME, considerando las complicaciones secundarias a dicha rama. No se encontró una diferencia estadísticamente significativa en la dosis acumulada de diuréticos entre ambas ramas.

\section{Comentarios}

General: El presente estudio pretendió establecer la eficacia del uso de $\mathrm{AH}$ a largo plazo como terapia en pacientes con cirrosis descompensada. Esto se justifica ante la falta de evidencia suficiente para determinar la efectividad y empleabilidad de la $\mathrm{AH}$ en este setting de pacientes.

Riesgo de sesgo: El diseño metodológico fue el adecuado para los objetivos del estudio. Fue randomizado con aleatorización computacional, con grupos sin diferencias significativas en variables demográficas, clínicas y de laboratorio. Tampoco hubo detención precoz del estudio. Una limitación destacable está dada por la ausencia de ciego en la asignación de tratamiento para pacientes, médicos y estadísticos. Además, aunque se estimaron 210 pacientes por rama, 83 pacientes se retiraron precozmente del estudio, por lo tanto, el análisis fue realizado por intención de tratar modificada. Finalmente, los pacientes del grupo TME $+\mathrm{AH}$ fueron evaluados de forma más frecuente por el equipo médico en el contexto de administración semanal de albúmina, lo que se traduce en un seguimiento más estricto con la consiguiente detección y manejo precoz de complicaciones.
Relevancia de los datos: Los datos resultan relevantes, ya que entregan evidencia sobre una potencial alternativa terapéutica disponible y costo-efectiva en el manejo de cirrosis descompensada.

Validez externa: Los criterios de inclusión y exclusión seleccionaron adecuadamente una población de pacientes que representa al grupo susceptible de requerir este tratamiento. Por otra parte, el estudio es de tipo pragmático, lo que implica que los protocolos implementados en el estudio podrían en teoría extrapolarse fácilmente al escenario clínico objetivo. En la misma línea de lo anterior, el grupo control representa de forma adecuada el manejo estándar que reciben los pacientes actualmente acorde a guías internacionales. Respecto a la $\mathrm{AH}$, resulta destacable que de los efectos adversos reportados no relacionados a cirrosis, ninguno de aquellos considerados grado 3 y 4 superó el $5 \%$ de frecuencia. La aplicación de esta terapia en nuestra realidad local podría verse limitada por el alto costo de $\mathrm{AH}$ en nuestro medio, así como por las dificultades que limitan su acceso en forma ambulatoria. Cabe recordar que este estudio no da cuenta de los costos no relacionados a la atención en salud o indirectos, elementos que deben ser considerados en futuros análisis de costo-efectividad.

\section{Conclusiones}

La administración de albúmina semanal podría disminuir la mortalidad en pacientes con cirrosis descompensada. Además, su administración optimiza el manejo de la ascitis en este grupo de pacientes, disminuyendo la estadía hospitalaria.

\section{Referencias}

1.- Caraceni P, Riggio O, Angeli P, Alessandria C, Neri S, Foschi F, et al. Long-term albumin administration in decompensated cirrhosis (ANSWER): an open-label randomized trial. Lancet. 2018;391:2417-29.

2.- Caraceni P, Riggio O, Angeli P, Alessandria C, Neri S, Foschi FG, et al. Long-term albumin administration in decompensated cirrhosis (ANSWER): an open-label randomized trial. Lancet 2018; publicado en línea 31 de mayo.

3.- Tsochatzis EA, Bosch J, Burroughs AK. Liver cirrhosis. Lancet. 2014;383:174961.

4.- Henriksen JH, Stage JG, Schlichting
P, Winkler K. Intraperitoneal pressure: ascitic fluid and splanchnic vascular pressures, and their role in prevention and formation of ascites. Scand J Clin Lab Invest 1980;40:493-501.

5.- Stepanova M, De Avila L, Afendy M, Younossi I, Pham H, Cable R, et al. Direct and indirect economic burden of chronic liver disease in the United States. Clin Gastroenterol Hepatol 2017; 15:75966.

6.- D'Amico G, Garcia-Tsao G, Pagliaro L. Natural history and prognostic indicators of survival in cirrhosis: a systematic review of 118 studies. J Hepatol 2006;44:217-31.

7.- Bernardi M, Moreau R, Angeli P, Schnabl B, Arroyo V. Mechanisms of decompensation and organ failure in cirrhosis: From peripheral arterial vasodilation to systemic inflammation hypothesis. J Hepatol 2015;63: 1272-84.

8.- Liumbruno G, Bennardello F, Lattanzio A, Piccoli P, Rossetti G. Recommendations for the use of albumin and immunoglobulins. Blood Transfus 2009;7:216-34.

9.- García-Martínez R, Caraceni P, Bernardi M, Gines P, Arroyo V, Jalan R. Albumin: pathophysiologic basis of its role in the treatment of cirrhosis and its complications. Hepatology 2013;58:1836-46.

10.- Gentilini P, Casini-Raggi V, Di Fiore G, Romanelli RG, Buzzelli G, Pinzani M, 
et al. Albumin improves the response to diuretics in patients with cirrhosis and ascites: results of a randomized, controlled trial. J Hepatol 1999;30:63945.

11.- Romanelli RG, La Villa G, Barletta G, Vizzutti F, Lanini F, Arena U, et al. Longterm albumin infusion improves survival in patients with cirrhosis and ascites: an unblinded randomized trial. World J Gastroenterol 2006;12:1403-7.

12.- European Association for the Study of the Liver. EASL clinical practice guidelines on the management of ascites, spontaneous bacterial peritonitis, and hepatorenal syndrome in cirrhosis. J Hepatol 2010;53:397-417.

13.- Runyon BA, AASLD. Introduction to the revised American Association for the Study of Liver Diseases Practice Guideline management of adult patients with ascites due to cirrhosis 2012. Hepatology 2013;57:1651-53.

14.- Moore KP, Wong F, Gines P, Bernardi M, Ochs A, Salerno F, et al. The management of ascites in cirrhosis: report on the consensus conference of the International Ascites Club. Hepatology 2003;38:258-66.

15.- Szende A, Janssen B, Cabases J, eds. Self-reported population health: an international perspective based on EQ5D. Dordrecht: Springer, 2014.

16.- Scalone L, Cortesi PA, Ciampichini R, Belisari A, D'Angiolella LS, Cesana G, et al. Italian population-based values of EQ-5D health states. Value Health 2013;16:814-22. 